\title{
NOVAS PERSPECTIVAS EM COMUNICAÇÃO SUPLEMENTAR E/OU ALTERNATIVA A PARTIR DA ANÁLISE DE PERIÓDICOS INTERNACIONAIS
}

\author{
New perspectives in augmentative and alternative communication \\ in international journal analysis
}

\author{
Carla Ciceri Cesa ${ }^{(1)}$, Ana Paula Ramos-Souza (2), Themis Maria Kessler ${ }^{(3)}$
}

\begin{abstract}
RESUMO
Tema: a Comunicação Suplementar e/ou Alternativa (CSA) é uma área do conhecimento da prática clínica, educacional e de pesquisa que favorece a qualidade de vida de seus usuários e familiares. Objetivo: propor diretrizes de intervenção e pesquisa na área de CSA a partir da identificação e síntese descritiva de artigos publicados em periódicos indexados em bases de dados eletrônicas internacionais, quanto à temática comunicação suplementar e/ou alternativa, abrangendo pesquisas experimentais, relatos de atendimento individual e/ou em grupo, estudos de casos que incluíssem sujeitos com paralisia cerebral ou com problemas neurológicos não definidos e/ou com retardo mental. Conclusão: A individualização das práticas quanto ao design, relevância e velocidade do processamento da informação e o processo de inclusão da família e demais parceiros conversacionais são fundamentais ao sucesso na intervenção, generalização e manutenção de uso da prancha de comunicação suplementar e/ou alternativa em contextos formais e informais. A prescrição individualizada dos recursos da área de CSA conforme características físicas, mentais, psíquicas, cognitivas e lingüísticas do usuário tem impacto positivo na sua qualidade de vida e na de seus familiares. Portanto, recomenda-se a introdução o mais breve possível dos recursos terapêuticos da área da comunicação suplementar e/ou alternativa para sujeitos com oralidade restrita e/ou ausente.
\end{abstract}

DESCRITORES: Linguagem; Paralisia Cerebral; Comunicação

\section{INTRODUÇÃO}

A Comunicação Suplementar e/ou Alternativa (CSA) tem-se apresentado como importante área

(1) Fonoaudióloga; Docente do curso de Fonoaudiologia da Faculdade Nossa Senhora de Fátima, Caxias do Sul, RS; Docente do curso de Pós-Graduação do Instituto Superior de Educação Ivoti, Instituto de Desenvolvimento Social Brava Gente, Porto Alegre, RS; Terapeuta domiciliar em Porto Alegre, RS; Mestre em Distúrbios da Comunicação Humana pela Universidade Federal de Santa Maria.

(2) Fonoaudióloga; Docente e Supervisora Clínica do curso de Graduação em Fonoaudiologia e do Programa de Pós-Graduação em Distúrbios da Comunicação Humana da Universidade Federal de Santa Maria, UFSM, Santa Maria, RS; Doutora em Letras pela Pontifícia Universidade Católica do Rio Grande do Sul.

(3) Fonoaudióloga; Docente, Supervisora Clínica e Coordenadora do curso de Graduação em Fonoaudiologia da Universidade Federal de Santa Maria, UFSM, Santa Maria, RS; Doutora em Letras pela Universidade Federal de Santa Maria.

Conflito de interesses: inexistente de conhecimento na prática clínica, educacional e de pesquisa, favorecendo sujeitos com impedimentos ou limitações para a produção oral de fala, de modo especial em quadros clínicos como a encefalopatia crônica infantil, mais comumentemente denominada de paralisia cerebral, a deficiência mental, dentre outros distúrbios de linguagem e desenvolvimento. Tal importância é evidenciada em um estudo demográfico realizado no estado norte-americano da Pensilvânia ${ }^{1}$, que identificou $12 \%$ de usuários de CSA na população pré-escolar, de diversas raças e etnias, e com vários distúrbios de linguagem e/ou desenvolvimento.

Apesar do considerável uso desse recurso na população infantil, ainda há pouca formação especializada sobre o tema no meio acadêmico. Esta afirmativa pode ser constatada em um estudo recente, de $2008{ }^{2}$, realizado através do envio de questionários para todos os centros de formação de terapeutas de linguagem e educadores especiais 
nos Estados Unidos da América (EUA). Os pesquisadores observaram que apenas $29 \%$ dos cursos possuem especialista no tema, embora $73 \%$ ofereçam ao menos um curso específico nesta área. Em relação à pesquisa, os foram encontradas apenas 120 teses e 55 dissertações. Apesar da afirmativa dos autores de que houve um crescimento percentual de estudo do tema naquele país, relacionado às novas exigências para a certificação clínica da American Speech-Language-Hearing Association (ASHA), ainda consideram deficitárias a difusão e as práticas laboratoriais na formação clínica especializada no tema (cerca de $50 \%$ dos cursos oferecem práticas em laboratório).

A realidade socioeconômica de países do primeiro mundo viabiliza recursos da área de CSA de alta tecnologia em tratamentos terapêuticos e educacionais, como se observa no programa assistencial do governo inglês, que assegura o acesso educacional e a intervenção clínica específica para fala e linguagem a sujeitos com necessidades especiais até os seus 19 anos, conforme aponta estudo realizado ${ }^{3}$.

Apesar dos limites socioeconômicos que dificultam o acesso à alta tecnologia assistiva em países em desenvolvimento, como o Brasil, é importante conhecer as possibilidades dessa tecnologia, assim como é fundamental saber explorar do melhor modo possível o uso de recursos de baixa tecnologia. Há diferentes tipos de pranchas de comunicação que variam desde baixa, média a alta tecnologia. Destaca-se que mesmo uma prancha de comunicação sendo de baixa tecnologia, não significa que tem pouca efetividade na sua função, que é a de proporcionar um espaço dialógico intersubjetivo do sujeito usuário deste recurso da área de CSA com o seu interlocutor.

Considerando, portanto, a necessidade de maior difusão acadêmica, clínica e educacional na área da CSA, este artigo se propõe a indicar diretrizes de intervenção e pesquisas a partir da identificação e síntese descritiva de artigos publicados em periódicos indexados em bases de dados eletrônicas internacionais da última década, quanto a temática CSA, abrangendo pesquisas experimentais, relatos de atendimento individual e/ou em grupo, estudos de casos que incluíssem sujeitos com paralisia cerebral ou com problemas neurológicos não definidos e/ou com retardo mental.

\section{MÉTODOS}

Para a realização da revisão de literatura foram realizadas buscas em bases de dados eletrônicas visando somente artigos científicos internacionais, por estes exporem um delineamento de pesquisa com critérios mais exigentes.

Os seguintes critérios iniciais de busca foram utilizados:

- Estudos no período de 1997 a 2008, faixa temporal em que houve maior difusão científica da CSA;

- Estudos publicados em periódicos indexados em bases de dados eletrônicas ( $A$ nacional a $A$ internacional): MEDLINE (via Bireme), SciELO, Cochrane e ASHA (Journal of Speech, Language and Hearing Research, American Journal of Speech-Language Pathology, e no Language, Speech and Hearing Services in Schools). Através da ASHA, foi possível ainda localizar diversos periódicos que, mesmo não sendo publicações suas, foram contemplados nesta busca: Communication Disorders Quarterly, The Journal of Rehabilitation, Topics in Language Disorders, Journal of Special Education, Topics in Early Childhood Special Education, Child: Care, Health and Development, Research and Practice for Persons with Severe Disabilities e o Applied Behavior Analysis.

Os unitermos escolhidos para a busca foram cerebral palsy, augmentative alternative communication, language therapy ou intervention.

Nesta primeira etapa resultaram um total de 319 artigos, os quais foram submetidos a um refinamento através de seleção manual (segunda etapa), levando-se em consideração os seguintes critérios de seleção: artigos que abordassem a inclusão de pesquisas experimentais, relatos de atendimento individual e/ou em grupo, estudos de casos que incluíssem sujeitos com paralisia cerebral ou com problemas neurológicos não definidos e/ou com retardo mental. Em uma terceira etapa, foi realizado outro refinamento manual, selecionando-se pesquisas que abordassem os processos de seleção, criação, confecção, introdução, intervenção, expansão (atualização do recurso), manutenção e generalização do uso do recurso da área da CSA em diferentes e, além disso que considerassem o foco no usuário e/ou neste com seus parceiros conversacionais (Tabela 1). Dessa consulta resultaram 26 artigos.

A partir da leitura dos 26 artigos selecionados, foram criadas duas categorias de análise, dividindoas em princípios e, destas, foram ainda realizadas subcategorizações, conforme descrito abaixo:

1. Princípios na seleção da CSA que abrangessem aspectos que relacionassem design e acessibilidade;

2. Princípios relacionados às estratégias terapêuticas de introdução, manutenção e generalização da CSA que contemplassem três 
Tabela 1 - Etapas de seleção dos periódicos

\begin{tabular}{lccc}
\hline Base de dados investigada & Primeira seleção & Segunda seleção & Terceira seleção \\
\hline MEDLINE & 114 & 29 & $\mathbf{0 2}$ \\
SciELO & 83 & 07 & 01 \\
Cochrane & 14 & 14 & 04 \\
ASHA & 108 & 32 & $\mathbf{1 9}$ \\
\hline TOTAL & 319 & 82 & $\mathbf{2 6}$ \\
\hline
\end{tabular}

subcategorias: estratégias focadas somente no usuário (2.1), estratégias centradas na relação entre o usuário e seus parceiros conversacionais, tais como família, professores, terapeutas, etc. (2.2) e aspectos linguísticos abordados na implementação dos recursos da área de CSA (2.3).

Dos 26 estudos selecionados, nove são estudos de grupo, sendo sete experimentais e dois descritivos, oito são de revisão de literatura, sete estudos de caso(s) e dois de formação profissional. Todos estes estão sintetizados na Figura 1.

\section{REVISÃO DA LITERATURA}

\section{Princípios na seleção da CSA: design e acessibilidade}

Nesta categoria, foram incluídos os artigos que abrangessem aspectos como o design do recurso para viabilizar a acessibilidade. Seis estudos indicam os principais aspectos encontrados:

- Intervenção inicial com crianças pequenas: a seleção direta do vocabulário é mais eficaz do que a varredura ${ }^{4} \mathrm{e}$, quando utilizada, deve ser animada ${ }^{5,6}$. O rastreamento com animação reduziu o número de sessões ${ }^{7}$, mas não deve ser utilizado na forma automática com crianças espásticas e não deve ser apresentado no design step (em níveis) para crianças com atetose ${ }^{8}$.

- Intervenção com adultos: um estudo ${ }^{9}$ demonstrou que o acesso ao teclado é melhor com atalhos via abreviaturas do que com o mouse.

- Considerando todas as faixas etárias, um estudo de revisão realizado ${ }^{8}$ afirma a necessidade de programação individual dos recursos tecnológicos tanto em relação à varredura quanto ao acesso e feedback. Quanto ao acesso à tela do monitor do computador (display), os autores recomendam preferencialmente o uso de cadeiras especiais, cunhas, bancos e/ou cavaletes do que o uso de um aparato adaptado denominado sidelyer, o qual posiciona a criança em decúbito lateral. Já quanto à iconicidade das simbologias gráficas, o referido estudo ${ }^{8}$ afirma ainda que o Pictogram Ideogram Communication (PIC) favorece mais a aquisição e generalização do que o BLISS e a ortografia.

\section{Estratégias terapêuticas de introdução, manutenção e generalização da CSA}

Um estudo de cunho geral ${ }^{10}$ nesta categoria aborda a revisão de 50 estudos, com 232 comparações, nas quais são analisadas as fases de intervenção, generalização e manutenção da CSA. Concluem que a intervenção e a generalização são mais bem sucedidas do que a manutenção, possivelmente porque muitos estudos têm foco menor na interação usuário-parceiro, como será visto nas próximas duas categorias.

\subsection{Estratégias focadas no usuário}

Observa-se que as estratégias focadas no usuário visam conjugar a introdução e uso dos recursos da área de CSA em contexto informal e formal, favorecendo, deste modo, o processo de generalização.

No contexto informal, o ensino do uso dos diversos tipos de recursos da área da CSA através de vídeos feitos em restaurantes de fast-food foi efetivo para que dois dos três alunos com deficiência de moderada a grave pudessem ter a capacidade de fazer pedidos após uma sessão. O terceiro aluno conseguiu após duas sessões ${ }^{11}$.

Em outro estudo considerando o mesmo contexto informal ${ }^{12}$, a CSA deu suporte e treinamento a seis sujeitos para que pudessem aumentar suas oportunidades de emprego. Os participantes melhoraram suas habilidades na busca de emprego, aprimoraram o uso de tecnologia de informação, permitindo lidar melhor com os seus distúrbios no ambiente de trabalho, o que se traduziu em aumento salarial em quatro dos seis sujeitos pesquisados.

Ao revisar a literatura sobre as práticas de ensino de tecnologia assistiva para bebês e crianças pequenas, foi constatado que, embora sejam encontrados resultados positivos, as evidências da efetividade do uso de recursos de tecnologia assis- 


\begin{tabular}{|c|c|c|c|}
\hline AUTORES/ANO & OBJETIVOS & MÉTODO & RESULTADOS E CONCLUSÕES \\
\hline $\begin{array}{l}\text { Schlosser e Lee } \\
(2000)\end{array}$ & $\begin{array}{l}\text { Determinar a efetividade } \\
\text { das intervenções em } \\
\text { comunicação aumentativa } \\
\text { e alternativa com uma } \\
\text { ênfase particular nas } \\
\text { estratégias que induzem } \\
\text { generalizações e/ou } \\
\text { manutenção. }\end{array}$ & $\begin{array}{l}\text { Analisaram } 50 \text { artigos selecionados } \\
\text { através de banco de dados eletrônico } \\
\text { dos últimos } 20 \text { anos. Foram realizadas } \\
232 \text { comparações de fases } \\
\text { experimentais, quantidade de } \\
\text { desfechos relatados e medidos. Os } \\
\text { estudos foram agrupados por } \\
\text { categorias: intervenção, generalização } \\
\text { e manutenção. }\end{array}$ & $\begin{array}{l}\text { A intervenção e a generalização são mais } \\
\text { bem sucedidas do que a manutenção. }\end{array}$ \\
\hline $\begin{array}{l}\text { Pennington e } \\
\text { McConachie } \\
(2001)\end{array}$ & $\begin{array}{l}\text { Testar a influência } \\
\text { comparativa de diversas } \\
\text { comorbidades cognitivas, } \\
\text { motoras e de } \\
\text { comunicação em crianças } \\
\text { com paralisia cerebral na } \\
\text { interação social. }\end{array}$ & $\begin{array}{l}\text { Foram avaliadas } 40 \text { crianças ( } 18 \\
\text { meninos e } 22 \text { meninas) com idade } \\
\text { entre } 2 \text { anos e } 8 \text { meses a } 10 \text { anos } \\
\text { através das variáveis comorbidades, } \\
\text { as quais foram correlacionadas com } \\
\text { medidas relacionadas a padrões de } \\
\text { interação. }\end{array}$ & $\begin{array}{l}\text { A inteligibilidade foi o maior fator preditivo } \\
\text { para a interação social. Os padrões de } \\
\text { conversação maternos não puderam ser } \\
\text { previstos, pois todas as mães usaram altos } \\
\text { níveis de iniciação e diretividade. Os } \\
\text { resultados indicam intervenções com foco } \\
\text { duplo, tanto nas crianças tanto nos } \\
\text { cuidadores. }\end{array}$ \\
\hline $\begin{array}{l}\text { Bedrosian, Hoag } \\
\text { e McCoy (2003) }\end{array}$ & $\begin{array}{l}\text { Análise dos efeitos da } \\
\text { relevância, velocidade e } \\
\text { características do juiz na } \\
\text { simulação entre usuário } \\
\text { de CSA e vendedor. }\end{array}$ & $\begin{array}{l}\text { Análise de } 20 \text { condições com a } \\
\text { manipulação de velocidade, relevância } \\
\text { e sexo dos juízes ( } 96 \text { vendedores). }\end{array}$ & $\begin{array}{l}\text { Lenta com aviso>lenta sem } \\
\text { aviso>parcialmente relevante> rápida sem } \\
\text { relevância. Os juízes valorizaram mais a } \\
\text { relevância da mensagem do que sua } \\
\text { velocidade neste estudo. }\end{array}$ \\
\hline $\begin{array}{l}\text { Drager et al. } \\
(2003)\end{array}$ & $\begin{array}{l}\text { Analisar três sistemas de } \\
\text { distribuição de } \\
\text { vocabulário: taxonômica, } \\
\text { esquemática e } \\
\text { contextualizado. }\end{array}$ & $\begin{array}{l}\text { Experimento com } 30 \text { crianças normais } \\
\text { com média de idade de } 2 \text { anos e } 6 \\
\text { meses expostas a três sistemas de } \\
\text { distribuição do vocabulário. }\end{array}$ & $\begin{array}{l}\text { O contexto do vocabulário é muito } \\
\text { importante para sua aquisição. }\end{array}$ \\
\hline $\begin{array}{l}\text { Lund e Light } \\
\text { (2003) }\end{array}$ & $\begin{array}{l}\text { Analisar a eficácia da } \\
\text { instrução gramatical para } \\
\text { usuários de CSA. }\end{array}$ & $\begin{array}{l}\text { Ensino de estruturas sintáticas pelo } \\
\text { uso da CSA a uma usuária de } 30 \text { anos } \\
\text { e um usuário de } 29 \text { anos. }\end{array}$ & $\begin{array}{l}\text { O foco sintático era sem contextualização. } \\
\text { Ambos aprenderam, mas um manteve } \\
\text { apenas por } 2 \text { meses e necessitou ser } \\
\text { retreinado. }\end{array}$ \\
\hline $\begin{array}{l}\text { Bryen, Cohen e } \\
\text { Carey (2004) }\end{array}$ & $\begin{array}{l}\text { Usar o Programa de } \\
\text { Suporte e Treinamento ao } \\
\text { Emprego Através da } \\
\text { Comunicação } \\
\text { Aumentativa (ACETS) } \\
\text { para aumentar as } \\
\text { oportunidades de } \\
\text { emprego de pessoas que } \\
\text { utilizam CSA. }\end{array}$ & $\begin{array}{l}\text { Foram incluídos seis sujeitos, e os } \\
\text { dados foram coletados através de uma } \\
\text { pesquisa sobre as habilidades } \\
\text { relacionadas ao emprego antes e após } \\
\text { a implementação do programa. Além } \\
\text { disso, foi utilizado um relatório on-line } \\
\text { de progresso ao longo do ano. }\end{array}$ & $\begin{array}{l}\text { Os participantes melhoraram suas } \\
\text { habilidades de busca de emprego, lidar } \\
\text { com o distúrbio no trabalho, comunicação e } \\
\text { tecnologia da informação. Além disso, } \\
\text { houve um impacto sobre o emprego e/ou } \\
\text { aumento salarial para quatro dos seis } \\
\text { participantes. }\end{array}$ \\
\hline Cress (2004) & $\begin{array}{l}\text { Descrever estratégias } \\
\text { com o foco na família } \\
\text { para implementar a CSA, } \\
\text { visando facilitar a } \\
\text { comunicação familiar. }\end{array}$ & $\begin{array}{l}\text { Revisão de artigos sobre intervenção } \\
\text { centrada na unidade de atendimento } \\
\text { família. Foram criados e analisados } \\
\text { contextos hipotéticos que retratam } \\
\text { situações cotidianas na relação } \\
\text { comunicacional. }\end{array}$ & $\begin{array}{l}\text { Adaptar individualmente a intervenção e ver } \\
\text { a família como unidade de serviço. }\end{array}$ \\
\hline $\begin{array}{l}\text { Drager et al. } \\
(2004)\end{array}$ & $\begin{array}{l}\text { Estudar o efeito do layout } \\
\text { da organização do menu. }\end{array}$ & $\begin{array}{l}\text { Experimento com } 30 \text { crianças normais } \\
\text { em que o vocabulário é apresentado } \\
\text { esquematicamente ou em cenas } \\
\text { contextualizadas. }\end{array}$ & $\begin{array}{l}\text { O vocabulário exposto em cenas } \\
\text { contextualizadas permitiu melhor } \\
\text { aprendizado e generalização a itens não } \\
\text { treinados. }\end{array}$ \\
\hline $\begin{array}{l}\text { Fallon et al. } \\
(2004)\end{array}$ & $\begin{array}{l}\text { Analisar a eficácia de um } \\
\text { programa de instrução de } \\
\text { leitura através de tarefas } \\
\text { de consciência fonológica } \\
\text { em usuários de CSA com } \\
\text { alterações neurológicas. }\end{array}$ & $\begin{array}{l}\text { Cinco sujeitos, com idades entre } 9 \text { e } \\
14 \text { anos, sendo três com retardo } \\
\text { mental e dois com paralisia cerebral, } \\
\text { realizaram tarefas fonológicas de } \\
\text { aliteração, busca de sons em outras } \\
\text { posições das palavras e leitura de } \\
\text { palavras com coda. }\end{array}$ & $\begin{array}{l}\text { Três demonstraram generalização para a } \\
\text { leitura via rota fonológica (palavras } \\
\text { desconhecidas). Quatro passaram a ler } \\
\text { livros, mas com dificuldade. Conclui-se que } \\
\text { é válida a aplicação do programa em } \\
\text { sujeitos com inteligibilidade de fala } \\
\text { prejudicada. }\end{array}$ \\
\hline Hoag et al. (2004) & $\begin{array}{l}\text { Análise dos efeitos de } \\
\text { conflito de máximas } \\
\text { conversacionais em } \\
\text { simulação entre usuário } \\
\text { de CSA e vendedor. }\end{array}$ & $\begin{array}{l}\text { Foram filmadas } 16 \text { condições em que } \\
\text { estavam em conflito a velocidade da } \\
\text { comunicação e a informatividade da } \\
\text { mensagem, sendo julgadas por } 96 \\
\text { juízes vendedores. }\end{array}$ & $\begin{array}{l}\text { A velocidade e a relevância se combinam } \\
\text { para produzir o melhor uso da CSA. }\end{array}$ \\
\hline
\end{tabular}




\begin{tabular}{|c|c|c|}
\hline AUTORES/ANO & OBJETIVOS & MÉTODO \\
\hline $\begin{array}{l}\text { Pennington, } \\
\text { Goldbart e } \\
\text { Marshall (2004) }\end{array}$ & $\begin{array}{l}\text { Revisar sistematicamente } \\
\text { todas as pesquisas } \\
\text { experimentais em } \\
\text { treinamento de comunicação } \\
\text { para parceiros } \\
\text { conversacionais de crianças } \\
\text { com paralisia cerebral e } \\
\text { avaliar a efetividade deste } \\
\text { tipo de intervenção. }\end{array}$ & $\begin{array}{l}\text { Análise de diversos bancos de dados } \\
\text { eletrônicos das áreas da saúde, } \\
\text { psicologia e educação até dezembro } \\
\text { de } 2002 \text {. Para serem incluídos para a } \\
\text { análise, os estudos teriam que relatar } \\
\text { intervenções com algum elemento de } \\
\text { controle experimental, com o objetivo } \\
\text { de mudar o estilo de interação dos } \\
\text { parceiros conversacionais de sujeitos } \\
\text { entre zero e } 19 \text { anos com paralisia } \\
\text { cerebral, mas sem graves } \\
\text { comprometimentos globais. }\end{array}$ \\
\hline
\end{tabular}

Starble et al. (2005)

Descrever uma abordagem desenvolver e implementar um treinamento de uso de recursos da área de CSA.

\begin{tabular}{ll}
\hline Bailey et al. & Examinar as percepções dos \\
(2006) & familiares em relação ao uso \\
& dos recursos da área de \\
& CSA
\end{tabular}
CSA. centrada na família para

\section{Foi realizado um treinamento com} uma família focando em avaliação das necessidades, identificação de contextos prioritários de comunicação implementação de recursos de CSA e treinamento do parceiro conversacional. Foi aplicado um questionário póstreinamento para avaliar a satisfação.

Foram realizadas entrevistas semiestruturadas com seis familiares de sete crianças que usam recursos da área de CSA para comunicação na escola.

\section{RESULTADOS E CONCLUSÕES}

Quatro estudos foram analisados, sendo que os seguintes procedimentos são positivos: cuidar o posicionamento dos parceiros conversacionais e das crianças na interação, criar oportunidades de comunicação e se dispor a fornecer responsividade aos sinais comunicativos da criança. Os estudos contêm falhas metodológicas, e os resultados podem não demonstrar que as mudanças são definitivamente um resultado da intervenção.

\section{Foram observados altos níveis de} satisfação para a maioria das dimensões (por exemplo: conhecimento e

sensibilidade do instrutor, relevância e adequação do treinamento). Outras dimensões foram associadas à satisfação menor, como, por exemplo, o conforto da família ao usar o recurso de CSA.

Os dados coletados foram agrupados em quatro categorias: expectativas, facilitadores, barreiras e benefícios do uso de recursos da área de CSA. Muito estresse está relacionado com o tempo de uso do recurso e, por isso, é preferido o uso de comunicação corporal. A prática e uso devem ser centrados na família.

\begin{tabular}{lll}
\hline Binger e Light & $\begin{array}{l}\text { Realizar um levantamento } \\
\text { demográfico acerca do perfil }\end{array}$ & $\begin{array}{l}\text { Para obter estas informações, uma } \\
\text { pesquisa foi desenvolvida e distribuída } \\
\text { de pré-escolares que utilizam }\end{array}$ \\
$\begin{array}{ll}\text { em pré-escolas de educação especial } \\
\text { o recurso da CSA. }\end{array}$ & $\begin{array}{l}\text { no estado norte-americano da } \\
\text { Pensilvânia. }\end{array}$
\end{tabular}

O estudo evidenciou $12 \%$ de usuários de CSA na população pré-escolar, de diversas raças e etnias e com vários distúrbios de linguagem e/ou desenvolvimento. Os achados indicam a necessidade urgente de todas as préescolas terem especialistas em fala e linguagem preparados para oferecer serviços para crianças que requerem CSA.

\begin{tabular}{|c|c|c|}
\hline Campbell (2006) & $\begin{array}{l}\text { Revisar a literatura sobre } \\
\text { práticas de ensino para } \\
\text { crianças pequenas no uso de } \\
\text { recursos de tecnologia } \\
\text { assistiva. }\end{array}$ & $\begin{array}{l}\text { Revisão de } 23 \text { artigos sobre técnicas } \\
\text { de ensino de tecnologia assistiva } \\
\text { publicados entre } 1980 \text { e } 2004 \text {, com } \\
\text { foco em bebês e crianças pequenas. }\end{array}$ \\
\hline et al. & $\begin{array}{l}\text { Analisar a aprendizagem de } \\
\text { códigos de abreviação e } \\
\text { expansão associados a itens } \\
\text { específicos de vocabulário } \\
\text { com acesso pelo mouse e } \\
\text { teclado. }\end{array}$ & $\begin{array}{l}\text { Experimento com } 30 \text { adultos normais } \\
\text { no qual foram apresentados os } \\
\text { códigos de abreviação e expansão } \\
\text { associados ao vocabulário com } \\
\text { acesso pelo mouse e teclado. }\end{array}$ \\
\hline $\begin{array}{l}\text { Harty, Alant e } \\
\text { Uys (2006) }\end{array}$ & $\begin{array}{l}\text { Descrever as crenças de } \\
\text { auto-eficácia materna e } \\
\text { classificação por parte da } \\
\text { mãe das habilidades } \\
\text { linguísticas de seus filhos. }\end{array}$ & $\begin{array}{l}\text { Vinte e cinco mães de sujeitos com } \\
\text { distúrbios da comunicação } \\
\text { completaram questionário sobre auto- } \\
\text { eficácia e classificação materna das } \\
\text { habilidades linguísticas da criança. }\end{array}$ \\
\hline
\end{tabular}

Embora haja resultados positivos, as evidências da efetividade do uso de recursos de tecnologia assistiva são limitadas em termos de número de relatos, áreas de conteúdo e nível de evidências.

Os resultados demonstraram que 0 acesso ao teclado permite maior domínio dos códigos de abreviação do que o mouse.

Os resultados demonstram que as mães acreditam ter alta auto-eficácia, embora os filhos tenham dificuldades de comunicação. Entre as funções avaliadas, as que apresentaram menor competência foram as de disciplinar e ensinar.

Johnston (2006) Os objetivos do estudo foram: Revisão da literatura para verificar se a) examinar a importância da a adequação contextual é relevante adequação contextual no delineamento e implementação da intervenção em CSA; b) explorar o potencial para o desenvolvimento e implementação do sistema CSA.

Aumentar a eficiência do CSA para usuários e parceiros aumenta a adequação contextual e, por consequência, aumenta a efetividade global das intervenções em CSA. da função da eficiência nas respostas para aprimorar a adequação contextual; c) apresentar uma estrutura para examinar a eficiência nas respostas da intervenção em CSA. 


\begin{tabular}{|c|c|c|c|}
\hline AUTORES/ANO & OBJETIVOS & MÉTODO & RESULTADOS E CONCLUSÕES \\
\hline $\begin{array}{l}\text { MacCarthy et al. } \\
\text { (2006) }\end{array}$ & $\begin{array}{l}\text { Comparar o rastreamento } \\
\text { com e sem animação. }\end{array}$ & $\begin{array}{l}\text { Vinte crianças foram testadas quanto } \\
\text { ao rastreamento com e sem } \\
\text { animação. }\end{array}$ & $\begin{array}{l}\text { Contextualização via animação facilita a } \\
\text { compreensão e uso da CSA. }\end{array}$ \\
\hline $\begin{array}{l}\text { Mechling e } \\
\text { Cronin (2006) }\end{array}$ & $\begin{array}{l}\text { Ensinar alunos com } \\
\text { distúrbios intelectuais de } \\
\text { moderado a grave a fazer } \\
\text { o pedido em restaurantes } \\
\text { fast-food usando recursos } \\
\text { de CSA. }\end{array}$ & $\begin{array}{l}\text { A intervenção consistiu da aplicação } \\
\text { de instruções em vídeo com o uso do } \\
\text { computador em três estudantes do } \\
\text { ensino médio. }\end{array}$ & $\begin{array}{l}\text { Para dois dos três alunos, a capacidade de } \\
\text { fazer pedidos aumentou drasticamente } \\
\text { imediatamente após a intervenção. } O \\
\text { terceiro aluno melhorou após uma segunda } \\
\text { aplicação. }\end{array}$ \\
\hline $\begin{array}{l}\text { Millar, Light e } \\
\text { Scholosser } \\
\text { (2006) }\end{array}$ & $\begin{array}{l}\text { Análise da efetividade da } \\
\text { CSA e seu impacto na } \\
\text { fala em estudos de } 1975 \\
\text { a } 2003 \text {. }\end{array}$ & $\begin{array}{l}\text { Analisar } 23 \text { estudos envolvendo } 67 \\
\text { sujeitos. }\end{array}$ & $\begin{array}{l}\text { A CSA favoreceu a fala em } 55 \text { dos } 67 \\
\text { sujeitos analisados. Apenas em } 5 \text { em casos } \\
\text { a fala decaiu. Há percepção de que a fala é } \\
\text { favorecida pela CSA. }\end{array}$ \\
\hline $\begin{array}{l}\text { Schlosser e } \\
\text { Sigafoos (2006) }\end{array}$ & $\begin{array}{l}\text { Analisar a efetividade da } \\
\text { CSA com e sem suporte } \\
\text { ou ambos. }\end{array}$ & Analisaram 45 estudos. & $\begin{array}{l}\text { O treino experimental pode ser uma forma } \\
\text { de escolher. O desfecho demonstra ganhos } \\
\text { com a CSA. Mais verificações para } \\
\text { observar eficácia. Há falhas metodológicas } \\
\text { nos estudos que acabam por prejudicar } \\
\text { conclusões. }\end{array}$ \\
\hline $\begin{array}{l}\text { Wagner e } \\
\text { Jackson (2006) }\end{array}$ & $\begin{array}{l}\text { Comparar os efeitos da } \\
\text { seleção direta e da } \\
\text { varredura na memória do } \\
\text { vocabulário. }\end{array}$ & $\begin{array}{l}\text { Experimento com } 120 \text { crianças em } \\
\text { aquisição típica em idade pré-escolar. }\end{array}$ & $\begin{array}{l}\text { Os resultados indicaram que o uso da } \\
\text { seleção direta é mais adequado do que a } \\
\text { varredura em tela fixa para crianças } \\
\text { pequenas. }\end{array}$ \\
\hline $\begin{array}{l}\text { Pennington, } \\
\text { Goldbart e } \\
\text { Marshall (2007) }\end{array}$ & $\begin{array}{l}\text { Investigar a efetividade } \\
\text { dos tratamentos da fala e } \\
\text { linguagem oferecidos às } \\
\text { crianças com paralisia } \\
\text { cerebral que apresentam } \\
\text { diferentes tipos e } \\
\text { gravidades de transtornos } \\
\text { de comunicação. }\end{array}$ & $\begin{array}{l}\text { Revisão sistemática exploratória dos } \\
\text { estudos publicados em banco de } \\
\text { dados eletrônicos até } 2002 \text {. Foram } \\
\text { selecionados } 12 \text { estudos, mas dois } \\
\text { deles abordavam os mesmos dados, } \\
\text { sendo descartado um deles. }\end{array}$ & $\begin{array}{l}\text { Os resultados da análise dos sete estudos } \\
\text { das crianças mostram aspectos que devem } \\
\text { ser valorizados na intervenção direta, tais } \\
\text { como habilidades de comunicação pré- } \\
\text { intencional (fixar o olhar, a expectativa dos } \\
\text { comportamentos em rotinas familiares, } \\
\text { etc.), funções pragmáticas e as estruturas } \\
\text { da linguagem expressiva. Os resultados } \\
\text { dos quatro estudos centrados na relação } \\
\text { pais ou outros parceiros conversacionais } \\
\text { com usuários crianças também apontam } \\
\text { êxito neste tipo de intervenção, } \\
\text { demonstrado através das mudanças de } \\
\text { seus estilos de interação (frequência de } \\
\text { perguntas abertas, proporção de perguntas } \\
\text { e respostas, etc.) Apesar dos desfechos } \\
\text { serem positivos, há falhas no tratamento } \\
\text { metodológico, indicando que não é possível } \\
\text { concluir que a intervenção é mais efetiva do } \\
\text { que a falta desta. }\end{array}$ \\
\hline $\begin{array}{l}\text { Saito e Turnbull } \\
(2007)\end{array}$ & $\begin{array}{l}\text { Analisar desfechos e } \\
\text { perspectivas familiares } \\
\text { em relação à prática de } \\
\text { CSA para identificar } \\
\text { questões que as famílias } \\
\text { percebem como } \\
\text { importantes para suas } \\
\text { vidas. }\end{array}$ & $\begin{array}{l}\text { Revisão de } 13 \text { artigos publicados entre } \\
1985 \text { e } 2005 \text {, que abrangessem } \\
\text { sujeitos de até } 21 \text { anos. Utilizou-se } \\
\text { uma escala de medição de Qualidade } \\
\text { de Vida Familiar (QVF) para avaliar os } \\
\text { estudos. }\end{array}$ & $\begin{array}{l}\text { O impacto das práticas de CSA interage } \\
\text { com cinco domínios de Qualidade de Vida } \\
\text { Familiar: interação familiar, função parental, } \\
\text { bem-estar físico e material, suporte em } \\
\text { relação ao distúrbio e bem-estar emocional. } \\
\text { A família deve ser o foco da prática e da } \\
\text { pesquisa. }\end{array}$ \\
\hline $\begin{array}{l}\text { Ratcliff, Koul e } \\
\text { Lloyd (2008) }\end{array}$ & $\begin{array}{l}\text { Realizar um levantamento } \\
\text { acerca da formação } \\
\text { especializada em CSA } \\
\text { nos Estados Unidos da } \\
\text { América. }\end{array}$ & $\begin{array}{l}\text { A pesquisa foi enviada para todos os } \\
\text { programas de treinamento de } \\
\text { especialistas em fala e linguagem via } \\
\text { e-mails direcionados aos diretores dos } \\
\text { programas ou das faculdades que } \\
\text { ensinam a CSA. }\end{array}$ & $\begin{array}{l}\text { Vinte e nove por cento dos cursos possuem } \\
\text { especialista no tema, embora } 73 \% \\
\text { ofereçam ao menos um curso específico } \\
\text { nesta área. É necessário ter mais } \\
\text { acadêmicos e clínicos preparados para a } \\
\text { área de CSA. }\end{array}$ \\
\hline
\end{tabular}

Legenda: CSA - Comunicação Suplementar e/ou Alternativa

\section{Figura 1 - Síntese dos artigos utilizados neste estudo}


tiva são limitadas em termos de número de relatos, áreas de conteúdo e nível de evidências ${ }^{13}$.

Além desses estudos, outros, que serão mencionados na seção 2.3, apresentam estratégias focadas no usuário ${ }^{5,14,15}$.

\subsection{Estratégias focadas na relação usuário-família e demais parceiros conversacionais}

Vários estudos apresentam consenso sobre a importância da inclusão familiar ${ }^{3,16-23}$ no processo terapêutico para que a generalização, ou seja, que o uso dos recursos da área de CSA seja efetivo fora do espaço terapêutico.

Em uma pesquisa de revisão ${ }^{16}$, foram analisados 13 artigos de 1985 a 2005, com sujeitos de até 21 anos, sendo observado, através de uma escala de qualidade de vida familiar (QVF), o impacto das práticas com recursos da área da CSA na QVF. Os autores observaram que cinco domínios revelam tal impacto: interação familiar, bem-estar físico e material, exercício da função parental, suporte ao distúrbio e promoção do bem-estar emocional ao usuário e familiares.

Outro artigo abordou estratégias focadas na família, a partir da criação de contextos hipotéticos de situações cotidianas na relação comunicacional entre profissionais, pais e sujeitos, concluindo que a intervenção deve ser adaptada individualmente, além de se considerar a família como unidade de serviço ${ }^{17}$.

Através de entrevistas semi-estruturadas com seis familiares de sete crianças usuárias de recursos da área de CSA, um estudo ${ }^{18}$ salientou que as expectativas, os facilitadores, as barreiras e os benefícios do uso do recurso devem ser analisados caso a caso com a participação familiar. Os autores afirmam que o excesso de estresse familiar está relacionado ao tempo de uso do recurso e, por isso, o uso de comunicação corporal é preferido ao uso dos recursos da área de CSA.

A abordagem centrada na família também é investigada em outro estudo ${ }^{19}$, visando desenvolver e implementar o uso dos recursos da área de CSA, resultando em altos níveis de satisfação da mesma com o conhecimento e sensibilidade do instrutor e relevância do treinamento, mas ainda algum desconforto para o uso do recurso, demonstrando que esta é uma questão para pesquisas futuras.

Pesquisadores afirmam que o foco duplo na criança e família, além de diferentes parceiros conversacionais, é o mais indicado, embora a efetividade das abordagens terapêuticas deva sofrer processo de aprimoramento considerando os critérios dos estudos baseados em evidências ${ }^{3,20,21}$. Do mesmo modo, estudos realizados demonstram que, apesar das esparsas evidências, o posicionamento corporal de parceiros conversacionais e crianças para a interação, a criação de oportunidades de comunicação e a responsividade aos sinais comunicativos pela criança são índices importantes a serem analisados durante o processo de intervenção ${ }^{3,21}$. De modo especial, as modificações que o parceiro pode fazer para facilitar o processo comunicativo, como frequência de perguntas abertas, proporção de perguntas e respostas, etc.

Outro estudo também corrobora a importância do foco no usuário em conjunto com o parceiro ao analisar a adequação contextual, pois a conversação e seu contexto verbal e não-verbal é um saber compartilhado pelos parceiros conversacionais ${ }^{22}$.

Por fim, para ilustrar a importância da unidade família na perspectiva de atuação com recursos da área da CSA, 25 mães de sujeitos com distúrbios da comunicação foram investigadas, visando descrever as crenças de auto-eficácia materna e classificação por parte da mãe das habilidades linguísticas de seus filhos ${ }^{23}$. Os resultados demonstram que as mães acreditam ter alta auto-eficácia, embora os filhos tenham dificuldades de comunicação.

\subsection{Aspectos linguísticos abordados na implementação dos recursos da área de CSA}

Em relação ao domínio de vocabulário, vários estudos demonstram que, do ponto de vista pragmático, a contextualização na apresentação do mesmo é fundamental para sua aquisição e generalização a outros espaços além do terapêutico, sobretudo no caso de crianças ${ }^{5-7}$.

Considerando a aquisição sintática, um estudo analisou a eficácia da instrução gramatical de categorias sintáticas distintas para dois adultos usuários dos recursos da área de CSA ${ }^{14}$. Ambos aprenderam, observando-se que um generalizou e outro necessitou ser retreinado para generalizar para contextos fora da sessão terapêutica.

Dada a relevância dos aspectos pragmáticos, pesquisas também foram realizadas nesta perspectiva de análise ${ }^{24,25}$. Em ambos os estudos, foi estudado o conflito entre máximas conversacionais de relevância (quantidade de informação relevante para a comunicação) e velocidade de veiculação da informação. Houve o julgamento de cenas, manipuladas com níveis distintos de ambas as variáveis, efetivado por 96 vendedores que visualizaram as situações de uso dos recursos da área de CSA durante compra e venda encenada por atores. Um estudo ${ }^{24}$ concluiu que a velocidade e a relevância devem se combinar para permitir o uso dos recursos da área de CSA, embora a velocidade fosse considerada mais importante pelos juízes nesse estudo. Já 0 outro estudo ${ }^{25}$, a relevância foi escolhida 
como fator mais importante. Nesse, a ordem de preferência de combinação entre velocidade e relevância na veiculação da mensagem foi: mensagem lenta e altamente relevante com aviso, seguida de mensagem lenta e altamente relevante sem aviso, posteriormente foi considerada mensagem rápida parcialmente relevante e, por fim, mensagem rápida sem relevância.

Em relação à aquisição da habilidade de falar, um artigo de revisão ${ }^{26}$ analisou 23 estudos, de 1975 a 2003, abrangendo 67 sujeitos e concluíram que, para 55 sujeitos, a fala foi favorecida pelo uso dos recursos da área de CSA, e decaiu apenas para cinco sujeitos. Em outro estudo ${ }^{20}$, as pesquisadoras afirmam que a inteligibilidade de fala do sujeito é fator preditivo para a interação social, observando que as mães procuram compensar tais dificuldades de interação através de altos níveis de iniciação e diretividade da conversação.

O uso do texto como unidade a ser considerada na intervenção é confirmado em outra pesquisa ${ }^{15}$ que afirma que o trabalho com palavras isoladas não produziu generalização para a leitura de textos. Neste estudo, cinco sujeitos, três com retardo mental e dois com paralisia cerebral, foram submetidos a programa centrado em tarefas de consciência fonológica. Três desenvolveram a rota fonológica de leitura e quatro passaram a ler livros com dificuldade.

Observa-se, ao término desta revisão, que é vasta a literatura científica sobre o design dos recursos da área de CSA, como iconicidade, cores e tamanhos de símbolos, disposição do layout, seleção, velocidade e demanda de esforço para acessar o recurso, além de diversas abordagens sobre a tecnologia assistiva de alta complexidade para viabilizar uma expressão comunicativa por parte do sujeito. Entretanto, ainda são em pequeno número as pesquisas clínicas ou experimentais na área de conhecimento da CSA na fonoaudiológica que valorizem a investigação e aplicação de práticas estratégicas na relação entre usuário e família (foco duplo) e demais parceiros conversacionais.

Realizada a revisão de literatura criou-se um esboço de hierarquização (Figura 2), apresentando uma síntese dos aspectos considerados mais relevantes para uma adequada implementação dos recursos da área de CSA, através dos processos de seleção, criação, confecção, introdução, intervenção, expansão, generalização e manutenção dos recursos da área da CSA.

Percebe-se, então, que os desafios para que se viabilize um uso realmente funcional dos recursos da área de CSA no cotidiano do usuário e demais parceiros conversacionais são de natureza variada.
Desta forma, criou-se quatro diretrizes para nortear o planejamento e execução destes processos:

1. Incluir o usuário, a família e demais parceiros conversacionais em todas as etapas, da seleção do design, do conteúdo linguístico e no processo de intervenção, para que ocorra o sucesso na generalização e manutenção do uso dos recursos da CSA em todos os espaços de vida do seu usuário. Para tanto, não só os familiares mais diretos (pais, irmãos, avós) como os menos diretos (tios, primos, etc.) e outros parceiros (colegas, professores, demais terapeutas, prestadores de serviços) devem ser incluídos;

2. Atentar ao impacto do recurso na qualidade de vida do usuário e da família. Para isso, devemse fomentar práticas clínicas nas quais as escoIhas dos recursos sejam compartilhadas, dialogadas com o próprio usuário e com a família, e não eleitas unilateralmente pelo terapeuta;

3. Considerar aspectos como velocidade de intercâmbio e a relevância da informação comunicativa na seleção de design e acessibilidade aos recursos da área de CSA e do conteúdo linguístico que ela veicula. Destaca-se que as temáticas linguísticas devem necessariamente atender às demandas temáticas do usuário e da família;

4. Favorecer o aprendizado do recurso da área de CSA e, consequentemente, sua manutenção e generalização conjugando contextos formais (escola, clínica) e informais (restaurantes, parque, vida social), assim como em contextos estruturados ou semi-estruturados de uso.

\section{CONCLUSÃO}

A individualização das práticas quanto ao design, relevância e velocidade do processamento da informação e o processo de inclusão da família e demais parceiros conversacionais são fundamentais ao sucesso na intervenção, generalização e manutenção de uso da prancha de comunicação suplementar e/ou alternativa em contextos formais e informais.

A prescrição individualizada dos recursos da área de CSA conforme características físicas, mentais, psíquicas, cognitivas e lingüísticas do usuário tem impacto positivo na sua qualidade de vida e na de seus familiares, portanto, recomendase a introdução o mais breve possível dos recursos terapêuticos da área da CSA para sujeitos com oralidade restrita e/ou ausente. 


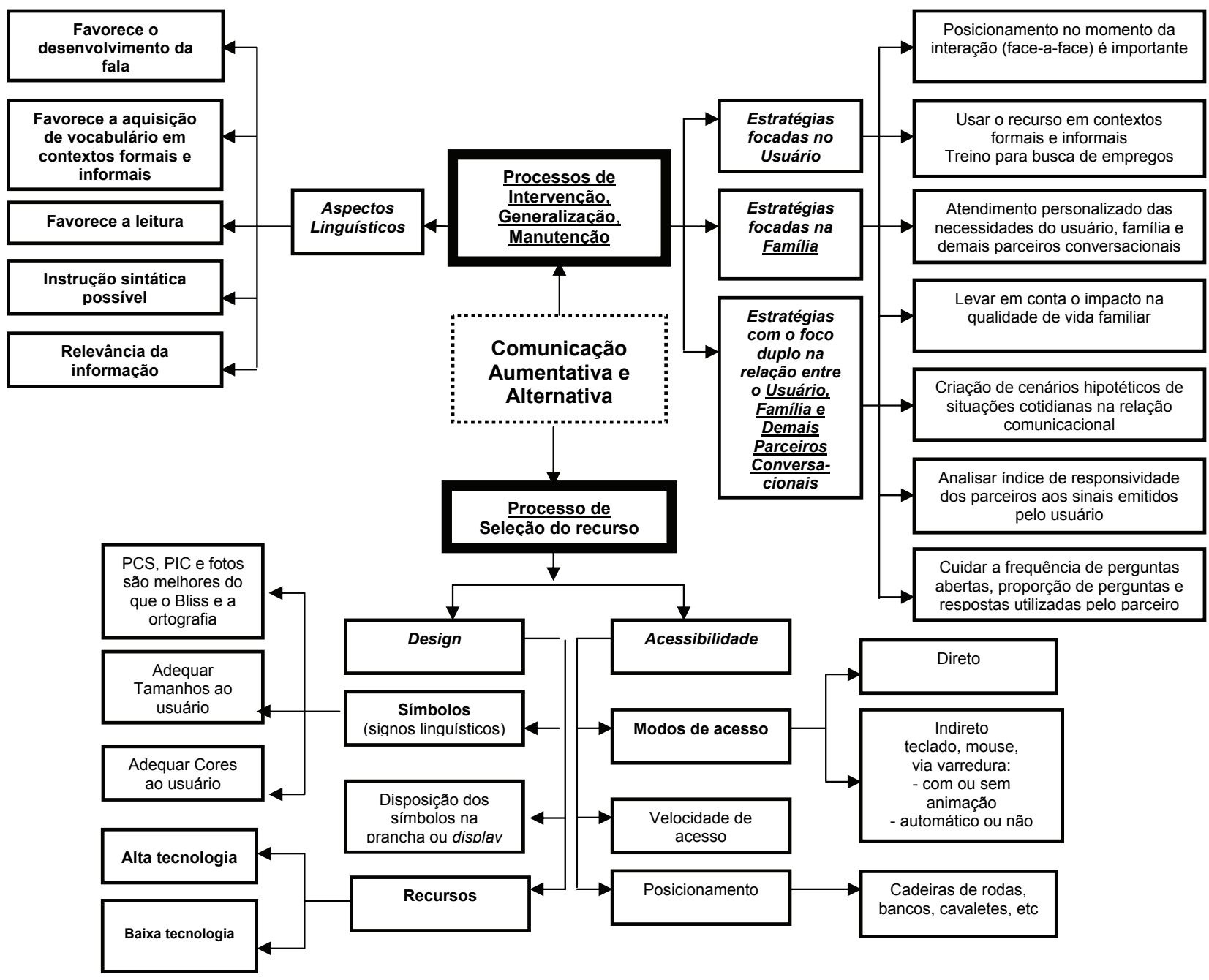

Figura 2 - Princípios da hierarquização para a implementação dos recursos da área de CSA

\begin{abstract}
Background: Augmentative and Alternative Communication (AAC) is a knowledge area of clinical, educational and research practice that favors the quality of life of its users and their relatives. Purpose: to propose intervention and research guidelines in AAC based on the identification and descriptive synthesis of articles published in indexed journals, in international electronic databases on AAC. Inclusion criteria were experimental research, reports of individual and/or group treatment, case studies, including subjects with cerebral palsy or with non-defined neurological diseases and mental retardation. Conclusion: individualization of the practices as for the design, relevance and speed of information processing and the process of including the family and other conversational partners are essential to a successful intervention, generalization and maintenance of AAC communication board within formal and informal contexts. Individual prescription of AAC resources according to physical, mental, psychic, cognitive and linguistic characteristics have a positive impact on the quality of life of users and their relatives. Therefore, it is recommended that AAC therapeutic resources are introduced as soon as possible for subjects with restricted and/or absent orality.
\end{abstract}

KEYWORDS: Language; Cerebral Palsy; Communication 


\section{REFERÊNCIAS}

1. Binger C, Light J. Demographics of preschoolers who require AAC. Lang Speech Hear Serv Sch. 2006; 37:200-9.

2. Ratcliff A, Koul R, Lloyd LL. Preparation in augmentative and alternative communication: an update for speech-language pathology training. J Speech Lang Pathol. 2008; 17:48-59.

3. Pennington L, Goldbart J, Marshall J. Tratamiento del habla y el lenguaje para mejorar las habilidades de comunicación de niños con parálisis cerebral. La Biblioteca Cochrane Plus [periódico on-line] 2007; (4):1-33. Oxford: Update Software [acesso em 10.05.2008]. Disponível em: URL: http://www.sld. cu/galerias/pdf/sitios/rehabilitacion-logo/terapia_ paralisis_cerebral.pdf

4. Wagner BT, Jackson HM. Developmental memory capacity resources of typical children retrieving picture communication symbols using direct selection and visual linear scanning with fixed communication displays. J Speech Lang Hear Res. 2006; 49:113-26.

5. Drager KDR, Light JC, Speltz JC, Fallon $K A$, Jeffries LZ. The performance of typically developing 2 1/2-year-olds on dynamic display AAC technologies with different system layouts and language organizations. J Speech Lang Hear Res. 2003; 46:298-312.

6. Drager KDR, Light JC, Carlson R, D'Silva K, Larsson B, Pitkin L, et al. Learning of dynamic display AAC technologies by typically developing 3-year-olds: effect of different layouts and menu approaches. J Speech Lang Hear Res. 2004; 47:1133-48.

7. McCarthy J, Light J, Drager K, McNaughton D, Grodzicki L, Jones J, et al. Re-designing scanning to reduce learning demands: the performance of typically developing 2-years-olds. Augment Altern Commun. 2006; 22(4):269-83.

8. Schlosser RW, Sigafoos J. Augmentative and alternative communication interventions for persons with developmental disabilities: narrative review of comparative single-subject experimental studies. Res Develop Disab. 2006; 27:1-29.

9. Gregory E, Soderman M, Ward C, Beukelman DR, Hux K. AAC Menu interface: effectiveness of active versus passive learning to master abbreviationexpansion codes. Augment Altern Commun. 2006; 22(2):77-84.

10. Schlosser RW, Lee DL. Promoting generalization and maintenance in augmentative and alternative communication: a meta-analysis of 20 years of effectiveness research. Augment Altern Commun. 2000; 16(4):208-26.
11. Mechling LC, Cronin B. Computer-based video instruction to teach the use of augmentative and alternative communication devices for ordering at fast-food restaurants. J Special Eduction. 2006; 39(4):234-45.

12. Bryen, DN, Cohen KJ, Carey A. Augmentative communication employment training and supports (ACETS): some employment-related outcomes. J Rehabil. 2004; 70(1):10-8.

13. Campbell PH, Milbourne S, Dugan LM, Wilcox MJ. A review of evidence on practices for teaching young children to use assistive technology devices. Top Early Child Spec Educ. 2006; 26(1):3-13.

14. Lund SK, Light J. The effectiveness of grammar instruction for individuals who use augmentative and alternative communication systems: a preliminary study. J Speech Lang Hear Res. 2003; 46:1110-23. 15. Fallon KA, Light J, McNaughton D, Drager K, Hammer $C$. The effects of direct instruction on the single-word reading skills of children who require augmentative and alternative communication. J Speech Lang Hear Res. 2004; 47:1424-39.

16. Saito $Y$, Turnbull A. Augmentative and alternative communication practice in the pursuit of family quality of life: a review of the literature. Res Prac Persons Severe Disabil. 2007; 32(1):50-65.

17. Cress CJ. Augmentative and alternative communication and language: understanding and responding to parents' perspectives. Top Lang Disord. 2004; 24(1):51-61.

18. Bailey RL, Parette Jr. HP, Stoner JB, Angell ME, Carroll K. Family members' perceptions of augmentative and alternative communication device use. Lang Speech Hear Serv Sch. 2006; 37:50-6.

19. Starble A, Hutchins T, Favro MA, Prelock $\mathrm{P}$, Bitner B. Family-centered intervention and satisfaction with AAC device training. Commun Disord Quart. 2005; 27(1):47-54.

20. Pennington L, McConachie H. Predicting patterns of interaction between children with cerebral palsy and their mothers. Dev Med Child Neurol. 2001; 43:83-90.

21. Pennington L, Goldbart J, Marshall J. Interaction training for conversational partners of children with cerebral palsy: a systematic review. Int $\mathrm{J}$ Lang Commun Dis. 2004; 39(2):151-70.

22. Johnston SS. Considering response efficiency in the selection and use of AAC systems. J SpeechLang Pathol Appl Behav Anal. 2006; 1(3):193-206.

23. Harty M, Alant E, Uys CJE. Maternal selfefficacy and maternal perception of child language competence in pre-school children with a communication disability. Child Care Health Dev. 2006; 33:144-54.

24. Hoag LA, Bedrosian JL, McCoy KF, Johnson $D E$. Trade-offs between informativeness and speed 
of message delivery in augmentative and alternative communication. J Speech Lang Hear Res. 2004; 47:1270-85.

25. Bedrosian JL, Hoag LA, McCoy KF. Relevance and speed of message delivery trade-offs in augmentative and alternative communication. J Speech Lang Hear Res. 2003; 46:800-18.
26. Millar DC, Light JC, Schlosser RW. The impact of augmentative and alternative communication intervention on the speech production of individuals with developmental disabilities: a research review. J Speech Lang Hear Res. 2006; 49:248-64.

DOI: 10.1590/S1516-18462010005000102

RECEBIDO EM: 22/08/2009

ACEITO EM: 31/05/2010

Endereço para correspondência:

Carla Ciceri Cesa

Av. Prof. Paula Soares, 315 ap. 302

Porto Alegre - RS

CEP: $91220-450$

E-mail: carlacesafga@yahoo.com.br

Rev. CEFAC. 2010 Set-Out; 12(5):870-880 